\title{
Stress distribution in the bonobo (Pan paniscus) trapeziometacarpal joint during grasping
}

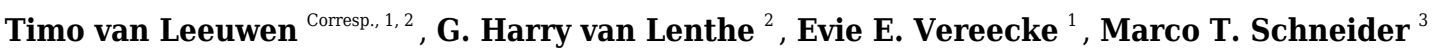 \\ ${ }^{1}$ Department of Development and Regeneration, KU Leuven, KULAK, Kortrijk, Belgium \\ 2 Department of Mechanical Engineering: Biomechanics section, KU Leuven, Leuven, Belgium \\ 3 Auckland Bioengineering Institute, University of Auckland, Auckland, New Zealand \\ Corresponding Author: Timo van Leeuwen \\ Email address: timo.vanleeuwen@kuleuven.be
}

The primate thumb plays a central role in grasping and the basal trapeziometacarpal (TMC) joint is critical to its function. The TMC joint morphology varies across primates, yet little is known about form-function interaction within in the TMC joint. The purpose of this study was to investigate how stress distributions within the joint differ between five grasping types commonly employed by bonobos (Pan paniscus). Five cadaveric bonobo forearms were CT scanned in five standardized positions of the hand as a basis for the generation of parametric finite element models to compare grasps. We have developed a finite element analysis (FEA) approach to investigate stress distribution patterns in the TMC joint associated with each grasp type. We hypothesized that the simulated stress distributions for each position would correspond with the patterns expected from a saddleshaped joint. However, we also expected differences in stress patterns arising from intraindividual variations in morphology. The models showed a high agreement between simulated and expected stress patterns for each of the five grasps ( $86 \%$ of successful simulations), while partially (52\%) and fully (16\%) diverging patterns were also encountered. We identified individual variations of key morphological features in the bonobo TMC joint that account for the diverging stress patterns and emphasized the effect of inter-individual morphological variation on joint functioning. This study gives unprecedented insight in the form-function interactions in the TMC joint of the bonobo and provides an innovative FEA approach to modelling intra-articular stress distributions, a valuable tool for the study of the primate thumb biomechanics. 


\section{Stress distribution in the bonobo (Pan paniscus) 2 trapeziometacarpal joint during grasping}

3

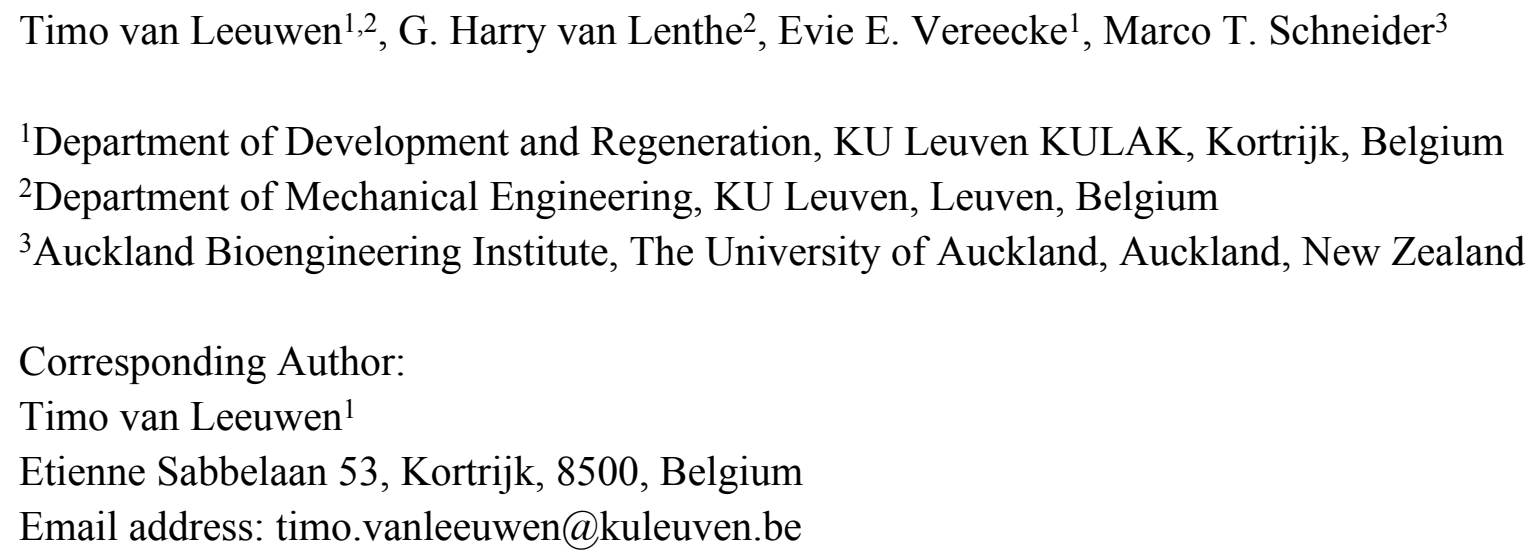

\section{Abstract}

The primate thumb plays a central role in grasping and the basal trapeziometacarpal (TMC) joint is critical to its function. The TMC joint morphology varies across primates, yet little is known about form-function interaction within in the TMC joint. The purpose of this study was to investigate how stress distributions within the joint differ between five grasping types commonly employed by bonobos (Pan paniscus). Five cadaveric bonobo forearms were CT scanned in five standardized positions of the hand as a basis for the generation of parametric finite element models to compare grasps. We have developed a finite element analysis (FEA) approach to investigate stress distribution patterns in the TMC joint associated with each grasp type. We hypothesized that the simulated stress distributions for each position would correspond with the patterns expected from a saddle-shaped joint. However, we also expected differences in stress patterns arising from intraindividual variations in morphology. The models showed a high agreement between simulated and expected stress patterns for each of the five grasps ( $86 \%$ of successful simulations), while partially $(52 \%)$ and fully $(16 \%)$ diverging patterns were also encountered. We identified individual variations of key morphological features in the bonobo TMC joint that account for the diverging stress patterns and emphasized the effect of inter-individual morphological variation on joint functioning. This study gives unprecedented insight in the formfunction interactions in the TMC joint of the bonobo and provides an innovative FEA approach to modelling intra-articular stress distributions, a valuable tool for the study of the primate thumb biomechanics. 
36

37

38

39

40

41

42

43

44

45

46

47

48

49

50

51

52

53

54

55

56

57

58

59

60

61

62

63

64

65

66

67

68

69

70

71

72

73

74

75

76

77

78

79

80

81

82

\section{Introduction}

The combination of high thumb mobility and forceful grasping is considered a unique modern human hallmark (Marzke 1997; Napier 1956, 1960; Napier and Napier 1967; Susman 1998). The high mobility of the modern human thumb predominantly originates from the morphology of the basal thumb or trapeziometacarpal (TMC) joint (Fig. 1) (Napier 1955). The TMC joint is a reciprocal saddle-shaped joint, the physiological equivalent of a universal joint which allows motion in two planes (flexion-extension, abduction-adduction) (Cooney and Chao 1977). The shape allows for large ranges of motion of the first metacarpal (MC1), but also offers the stability required for forceful grasping (D’Agostino et al. 2017; Napier 1955; Rose 1992). The morphology of the TMC joint varies across primates (Lewis 1977; Marzke et al. 2010; Rafferty 1990), but how this relates to specific thumb function in these primates remains unclear. Where many studies focus on the human TMC joint, especially from a clinical point of view (Crisco et al. 2015; Dourthe et al. 2016; Haines 1944; Kerkhof et al. 2018; Kuczynski 1974; Ladd et al. 2013, 2014; Schneider et al. 2015)., far less is known about form-function relations in the non-human primate thumb. In this study, we investigate the effect of different grip types on the stress distribution in their TMC joint in bonobos (Pan paniscus, Fam. Hominidea) (Fig. 2a). Bonobos are both arboreal and terrestrial, and they use their hands during locomotion and manipulation, including cases of tool use that have been observed in captivity. (Bardo et al. 2016; Christel, Kitzel, and Niemitz 1998; Crast et al. 2009; Feix et al. 2015; Ingmanson 1998; Jordan 1982; Neufuss et al. 2017). Common grasp types used by bonobos include various types of power grasps as well as a key pinch precision grip (Fig. 2) (Christel 1993; Christel et al. 1998; Napier 1956; Neufuss et al. 2017). In addition, bonobos use their thumb in vertical climbing and clambering, to grasp trunks or branches, but the thumb is not loaded during knuckle-walking (Inouye 1992; Samuel et al. 2018; Tuttle 1967; Wunderlich and Jungers 2009).

Bonobos, like humans, possess a saddle-shaped TMC joint, and have a very similar soft-tissue configuration, i.e. the surrounding muscles and ligaments, to that of humans (van Leeuwen et al. $2018,2019)$. Such morphology is not unique to hominids. In fact the saddle-shape is also present in New World monkeys (Lewis 1977; Rafferty 1990), some of which are also highly dextrous (Parker and Gibson 1977). In bonobos, the TMC joint is highly curved even when compared to humans (Marzke 1997), allowing for wide ranges of motion in particular during adduction and adduction of the thumb. Our previous research on the kinematics of the primate TMC joint has shown that the mobility of the bonobo thumb is similar to that of humans, but thumb extension is limited by the presence of a strong anterior oblique ligament which reinforces the palmar side of the TMC joint (van Leeuwen et al. 2019). Based on these findings, we expect that the force distribution within the TMC joint will reflect signals of the specific joint morphology. Recently, Schneider et al. (2017) developed a finite element (FE) approach to model stress distribution in the human TMC joint. Here, we build upon this work and propose a derived model-based method to investigate the stress distribution in the bonobo TMC joint.

In total, we investigate five types of grasps commonly used by bonobos (Christel 1993; Samuel et al. 2018). Out of these, three are large diameter power grasps, one with the thumb abducted, one with the thumb extended, and one with an adducted thumb. The abducted type, further referred to as powerL (Fig. 2b), is characterized by a wide grip and an abducted and externally rotated thumb that is still radioulnarly in line with the trapezium. Stresses are expected to be diverted to the radio-central aspect of the articular facet of the trapezium during powerL. The large

PeerJ reviewing PDF | (2021:01:57152:1:1:NEW 7 Jul 2021) 
83 diameter power grasp with extended thumb (powerLE) (Fig. 2c) extends the thumb near the 84 maximal excursion of the TMC joint, but in line with the dorsopalmar plane of the trapezium. Stress 85 peaks are therefore expected in the dorsal-central aspect of the articular facet of the trapezium. 86 The large diameter power grasp with thumb adducted (powerLA) (Fig. 2d) places the thumb in 87 line with the other digits of the hand. The thumb is adducted and externally rotated. Here, the 88 opposite of the grasps with abducted thumb is expected; high stress in the ulnar-central articular 89 facet of the trapezium. The small diameter power grasp (powerS) (Fig. 2e) is similar to the large 90 diameter variant, but in contrast, the amount of thumb abduction is less extreme. Stress is 91 expected to concentrate centrally on the articular facet, with a radial tendency. The key pinch (Fig. 2f) is characterized by a relatively straight thumb and trapeziometacarpal joint. External thumb tip force is exerted downwards towards the radial side of the middle phalanx of a flexed index finger. As a result, forces are expected to be diverted towards to the central-palmar aspect of the trapezial facet, creating stress peaks in this area. A visual representation of the expected stress patterns is included in the Results section (see Fig. 5, top row).

We simulate the stress distribution within the TMC joint during these five positions of the hand in five cadaveric bonobo forearms. We hypothesize that the stress distribution within the TMC joint for each individual grasp reflects the expected distribution for a universal joint during that grasp, as described above. Furthermore, we expect each individual stress distribution pattern to be influenced by the specimen's individual joint morphology. Therefore, we hypothesize that deviations from the predicted stress patterns for each grasp can be related to differences in TMC joint morphology.

\section{Materials \& Methods}

106

In this study, we use an FE approach to model TMC joint stress distributions during various types of grasps employed by bonobos in manipulation and arboreal locomotion. The focus of this study is on the distribution of stress within the TMC joint during each grasp, the localisation of stress peaks, and how stress distributions may be influenced by individual TMC joint morphology. We use an optimised parametric FE approach to model TMC joints of five cadaveric bonobo hands in five functional positions.

\section{Specimens}

114 Five cadaveric forearms of (sub)adult bonobos were obtained opportunistically via collaboration with five European zoos (Table 1). The forearms were disarticulated either at the glenohumeral joint or at the elbow joint and stored at $-18{ }^{\circ} \mathrm{C}$ shortly after decease. All specimens were part of parallel studies on bonobo forelimb morphology and thumb biomechanics (van Leeuwen et al. 2018, 2019). Specimen Pp2 was a sub-adult with a TMC joint that was not fully mature, presenting incomplete epiphysial fusion of the $\mathrm{MC1}$ and a relatively smooth articular periphery (without pronounced bony ridges). The specimen has been included in the sample as the articular surfaces were sufficiently developed to present the reciprocal saddle-shape of the adult TMC joint. No articular pathologies were observed at the TMC joint in any of the specimens used for this study. 
Each specimen was thawed at room temperature over a period of 24 hours before being imaged using a 64-slice Discovery HD 750 CT scanner [GE Healthcare, Little Chalfont, UK; display field of view (DFOV): $173 \mathrm{~mm}$; slice thickness: $0.625 \mathrm{~mm}$; pixel spacing: 0.338/0.338 mm, voxel size: $0.071 \mathrm{~mm}^{3} ; 100 \mathrm{kV} ; 180 \mathrm{~mA}$; image size: $512 \times 512$ pixels). A radio-translucent rig with a small $(50 \mathrm{~mm})$ and large diameter peg $(125 \mathrm{~m})$ was used to standardize each hand in the five functional grip positions (i.e. powerL, powerLA, powerLE, powerS, and pinch). This resulted in a total of 24 scan positions, as we were unable to position specimen Pp5 in a powerL grasp. Each trapezium and the first metacarpal was segmented separately using Mimics Research 20.0 (Materialise, Leuven, Belgium) and exported as triangular meshes.

\section{Parametric meshing}

Parametric FE meshes for 24 TMC joint models were generated in two steps by aligning all specimen-position combinations to a template set of TMC meshes and fitting the discretised master meshes to the 23 remaining specimen-position combinations.

137

Step 1: Mesh alignment

For each specimen, the trapezium and MC1 STL meshes of one specific grasp (pinch) were selected and designated as template meshes (Fig. 3 a). A master TMC set template mesh (Fig. 3a) was selected and reoriented to a TMC joint-based coordinate system where the principal directions of motion of the thumb (i.e. abduction-adduction, extension-flexion, and axial rotation) are aligned with the cartesian coordinate system using a method based on Halilaj et al. (2013; van Leeuwen et al., 2019). The individual template sets for the remaining specimens were aligned with the master mesh in the same coordinate system by rigid body registration fitting the trapezium to the master trapezium template, and transforming the corresponding MC1 (Fig. 3b). The MC1s of all remaining specimen-position combinations were aligned with their respective template trapezium by rigid body registration fitting each combination's respective trapezium and transforming the $\mathrm{MC} 1$ along the resulting rotation matrix. This yields five aligned triangular trapezium meshes, one per specimen, each with five corresponding triangular MC1 meshes, one per grasp (with the exception of Pp5, which has four) (Fig. 3c).

\section{Step 2: Mesh fitting}

The trapezium and MC1 master template triangular meshes were discretized into quadrilateral meshes using Instant Meshes software (Jakob et al. 2015) (Fig. 3d). The reciprocal subchondral articular surfaces of the TMC joint were identified on both bones from bone landmarks, and delineated via local meshing using Instant Meshes' Position Field and Orientation Field tools. To ensure the subchondral surface mesh quality, Instant Meshes' Singularity tools were used to reduce mesh irregularities, minimize element distortion and increase mesh Jacobian values (Burkhart, Andrews, and Dunning 2013). The delineated subchondral elements were extracted using MeshLab software (v2016.12) (Cignoni et al. 2008) and exported as separate quadrilateral meshes. Custom Python (3.6) scripts were used to generate quadrilateral template TMC meshes for each individual specimen. The discretised master trapezium, MC1, and their respective 
164

165

166

167

168

169

170

171

172

173

174

175

176

177

178

179

180

181

182

183

184

185

186

187

188

189

190

191

192

193

194

195

196

197

198

199

200

201

202

203

204

205

cartilage meshes were fitted to the vertices of each specimen's triangular template mesh using Radial Basis Function (RBF) fitting (Fig. 3e). A three-layer, hexahedral cartilage layer mesh was generated for each specimen by lofting the extracted subchondral meshes along the surface node normal using a custom Python script based on Schneider et al. (2017). As cartilage information for the bonobo TMC joint was lacking, the cartilage layer was generated at a thickness of $0.5 \mathrm{~mm}$, somewhat lower than the 0.6-0.7 mm thickness reported for human TMC joint cartilage (Dourthe et al. 2019) to account for the relatively smaller bone size. MC1 quadrilateral meshes for the remaining grasps of each specimen were generated by rigid body registration fitting their quadrilateral MC1 template mesh to each position (Fig. 3f), and using the resulting transformation matrices to transform the corresponding hexahedral cartilage meshes. This yields five quadrilateral trapezium meshes, one per specimen, each with five (with the exception of Pp5) corresponding quadrilateral MC1 meshes (Fig.3g), one per position, and hexahedral cartilage meshes for all bone meshes.

\section{FE model generation}

All FE models were generated and solved using FEBio Software Suite (Maas et al. 2012). A master model (Fig. 4) was manually set up in FEBio Preview (v2.1.3) to be solved by the FEBio solver (v.2.9.0). The quadrilateral bone surface meshes were modelled as rigid bodies, and connected to their respective hexahedral cartilage meshes, modelled as a neo-Hookean material (Young's modulus $=18 \mathrm{MPa}$, Poisson's ratio $=0.45)($ Black and Hastings 2013). The trapezium mesh was constrained in all degrees of freedom, while the MC1 was given three degrees of freedom, allowing it to translate but not rotate.

As the imaging was performed on cadaveric material, the resulting bone meshes were situated in unloaded positions. To account for this, the model was set up as a two-step, force-driven model. The first step serves to bring the elements into contact, facilitated by nonlinear springs, exerting $1 \mathrm{~N}$ of force each, corresponding to the bonobo TMC joint ligaments (van Leeuwen et al. 2019). Step two introduces the applied force vector. We performed a 2D static force analysis, based on Cooney and Chao (1977), to estimate the magnitude of the resultant force vector applied on the MC1. In the static force analysis, the initial thumb tip force was estimated at $50 \mathrm{~N}$, based on the physiological cross-sectional area of the thenar muscles, and muscle attachment information was based on detailed dissections published by our research group (van Leeuwen et al. 2018). The analysis yielded a force vector magnitude of about $300 \mathrm{~N}$, which was applied to the MC1 for all positions. Successful model termination was achieved when the FEBio solver was capable of executing all steps of the force application process without any elements collapsing.

The remaining 23 models were generated using a custom Python script that substitutes the master mesh data with the parametric mesh data and adapted the force vector orientation to conform to the first metacarpal orientation for each specimen-position combination.

To simulate the five grasping positions of the thumb, the models were optimized to bring the position after loading in close agreement with the scanned positions of the thumb. This was done using a Python-based function that optimizes the orientation of the force vector to yield a loaded terminating position that closely resembles the unloaded, scanned position of the MC1 by

Peerj reviewing PDF | (2021:01:57152:1:1:NEW 7 Jul 2021) 
206

207

208

209

210

211

212

213

214

215

216

217

218

219

220

221

222

223

224

225

226

227

228

229

230

231

232

233

234

235

236

237

238

239

240

241

242

243

244

245

246

reducing the root mean square (RMS) error between these positions. As RMSE gives a relatively high weight to larger errors, it helps guide the optimiser towards the optimal position of the MC1. The proximo-distal difference between the loaded and unloaded state was accounted for in the final RMSE calculation by excluding the articular gap distance.

Distal trapezial articular surface Von Mises stresses were normalized and plotted for each individual model. The location of stress peaks and the overall stress distributions were compared to the expected patterns for each position.

\section{Results}

Out of 24 simulated models, 21 terminated successfully. Two grips of Pp2 (powerL and powerS), and one grip of Pp5 (powerLA) were unable to successfully terminate using our method due to issues discussed below. Average RMS errors for the successfully terminated models were 1.364 $\mathrm{mm}$ for pinch, and below $0.7 \mathrm{~mm}$ for the power grasp simulations $(0.516 \mathrm{~mm}$ for powerL, 0.307 $\mathrm{mm}$ for powerLA, $0.514 \mathrm{~mm}$ for powerLE, and $0.667 \mathrm{~mm}$ for powerS).

Individual stress distribution plots (Fig. 5) show indications of the expected stress patterns in 18 out of the 21 simulations. During pinch, stress peaks are located centrally and palmarly when compared to the other positions in the same individual, cases of palmar edge loading occur in 3/5 individuals. During powerL, radial stress distributions are present in two-thirds of the simulations. During powerLA, forces are directed to the ulnar side in the four successful simulations. The powerLE position displays high stress on the dorsal aspect of the articulation in all five simulations. During powerS, forces are diverted to the far radial side in one simulation, to the central-radial side in another simulation, and to a combination of both the central and far radial aspect of the articular surface in a third.

Stress distributions diverging from the expected patterns are present in 14 simulations; 11 show divergent patterns in addition to the expected patterns, and three show divergent patterns only (Fig. 5). Pp1 shows divergent patterns on the ulnar aspect of the articular facet during pinch and powerS, as well as on the radial aspect during powerLA. Pp2 shows high stress peaks radiocentrally during all three successful positions; pinch, powerLA, and powerLE, but during powerLE it is part of the expected location. Pp3 shows divergent stress peaks on the dorso-ulnar aspect of the articular facet during pinch, powerL, and powerS, these peaks are also present during the other two positions, but in those cases they are part of the expected pattern. In addition, Pp3 shows divergent indications of contact on the radial side during powerLA. Pp4 shows stress peaks located ventrally on the articular surface in all five positions, although these are only expected during pinch. Stress distributions in Pp5 are directed ulnarly in all three of the successful simulations, but this is only unexpected during powers.

Peer) reviewing PDF | (2021:01:57152:1:1:NEW 7 Jul 2021) 


\section{Discussion}

248

249

250

251

252

253

254

255

256

257

258

259

260

261

262

263

264

265

266

267

268

269

270

271

272

273

274

275

276

277

278

279

280

281

282

283

284

285

286

287

288

289

290

The purpose of this study was to simulate functional grasps commonly used by bonobos and to investigate how stress distributions within TMC joint vary between these activities. We applied a computational modelling approach using parametric meshes to compare stress distributions across five individuals using five grasping tasks. The magnitude of the resultant force vectors were set to $300 \mathrm{~N}$ and the direction of the resultant force vectors were optimised in force-driven FE simulations for the 24 simulation cases. Of the 24 simulations, 21 simulations converged to an average RMSE of $0.673 \mathrm{~mm} \pm 0.364 \mathrm{~mm}$. We hypothesized that the simulated stress distributions for each position would correspond with the patterns expected from a saddle-shaped joint. However, we also expected there to be differences in stress patterns arising from individual variations in joint morphology.

Overall, the results appear to correspond with our predictions for each position, 21 of the 24 simulations showing stress distributions largely matching the expected distributions for a universal type joint. Out of these, there are 11 cases where additional patterns of stress distribution can be recognized, while the remaining three simulations show patterns fully diverging from the expected stress distributions. In summary, the results show that grip type influences the stress distributions in the expected manner, but variations in joint morphology interfere with this pattern. Most likely, morphological variations cause the joint to function less like a universal joint. A perfect universal joint consists of a double hinge that allows for rotation in two planes only, while restricting axial rotation (Cooney and Chao 1977). This strict functionality is by virtue of the shape of the joint and is lost when the morphology varies. In the current bonobo sample, such variations are prevalent and consequently, stress patterns appear to vary more between individuals than between grip types. A detailed interpretation of the individual differences is given below. Most of the deviations from the expected stress patterns can be explained by differences in joint morphology.

Our results give an indication of the influence of an individual's morphology on joint stress distribution (Fig. 5). The divergent patterns in Pp1 show high stress peaks on the dorsal aspect. Furthermore, there are indications of palmar contact during powerLE and powerS. When inspecting the TMC joint morphology of $\mathrm{Pp} 1$, these patterns coincide with the relatively protruding dorsal ridge and palmar beak of its MC1 (Fig. 6a), which come in contact with the dorsal and palmar parts of the articular surface of the trapezium upon loading. Pp2 shows stress peaks in the central-radial aspect of the articular surface in all three successful positions, while it was only expected in powerLA. This may be attributed to the dorsal ridge of its MC1 articular surface, which is more asymmetrical than in the other specimens. In Pp2, the most proximal point of the dorsal ridge is slightly offset to the radial side of facet. This causes contact with the trapezium, which symmetrical shape does not accommodate for the offset protrusion (Fig. 6b). During pinch, the asymmetrical dorsal ridge aspect may initiate contact before the palmar beak. Furthermore, Pp2 is a sub-adult with a TMC joint that is not fully mature, resulting in a smoother articular periphery and in particular less pronounced dorsal and palmar ridges. . This affects the shape of the TMC articular surfaces and appears to influence the interaction between the palmar beak and palmar aspect of the trapezium during pinch particularly, possibly causing it to not show the expected stress peaks in the palmar region during this grasp. Pp3 shows relatively high average stress

PeerJ reviewing PDF | (2021:01:57152:1:1:NEW 7 Jul 2021) 
291

292

293

294

295

296

297

298

299

300

301

302

303

304

305

306

307

308

309

310

311

312

313

314

315

316

317

318

319

320

321

322

323

324

325

326

327

328

329

330

331

332

333

334

peaks on the dorso-ulnar aspect of the articular facet in all positions. Upon visual assessment of this specimen it is clear that its joint morphology is relatively robust compared to the other specimens, i.e. the morphological features of the trapezium and MC1 are more strongly developed. The dorso-ulnar periphery of the trapezium's distal facet protrudes heavily (Fig. 6c), interacting with the MC1 in every modelled scenario. All positions collide heavily on the ulnar aspect of the facet, causing high stress in that area for each position. Two of the three divergent patterns (that do not also show an expected pattern) are Pp3's powerL and powerS, which are both power grips with the thumb abducted. These two grips show the same divergent stress patterns. It appears that in these cases, the MC1 loads the protruding dorso-ulnar aspect of the trapezial facet's periphery instead of initiating contact on the radial side of the trapezial articular surface as expected for these grasps. This causes the protrusion to function as a 'bony stop', for which bone is not intended (Simpson, Latimer, and Lovejoy 2018). Such loading of the articular surface periphery may be the result of a self-perpetuating cycle where the high loads cause inflammation and pathological bone responses. Despite the absence of osteophytes, the protrusion may be an early sign of osteoarthritis (Wilson, McWalter, and Johnston 2008), although reports of hand osteoarthritis are rare in non-human primates. Also during pinch, high stresses are observed in the dorso-ulnar region, which can be explained by the protrusion on the articular periphery. Pp3's divergent stress patterns further show loading on the radial aspect of the trapezial articular facet during powerLA, powerLE, and powerS, as well as on the palmar edge during powerLA. This is likely due to the pronounced dorsal ridge and palmar beak on the MC1. The divergent stress patterns in Pp4 indicate loading on the palmar aspect of the articular facet. The trapezial morphology shows that the palmar aspect of the articular surfaces flattens out relatively fast towards the palmar aspect (Fig. 6d). At the bottom, the surface shows light indications of indenture, which may capture the palmar beak during loaded positions (Fig. 6d). Pp5 shows divergent stress patterns on the ulnar side of the trapezial facet. This appears to be caused by a protrusion of the trapezial facet's ulnar periphery, as seen in Pp3 (Fig. 6c). The protrusion, however, is not as explicit as in the case of Pp3, which may explain the lower stress peaks in Pp5.

The bonobo is a primate species underrepresented in literature. Due to its remote and scarce population (Prüfer et al. 2012), academic focus on the bonobo has been limited in in comparison to its sister taxon; the chimpanzee (Pan troglodytes). Studies on the functional anatomy of the bonobo remain limited (Miller, 1952; Vereecke et al., 2005; Payne et al., 2006a; Payne et al., 2006b; Myatt et al., 2012; Diogo et al., 2017), and most literature including Pan only make use of chimpanzees (Lewis 1977; Marzke et al. 2010; Rafferty 1990; Rose 1992). Claims have been made that due to evolutionary stasis, the bonobo may be the most suitable model for the Pan Homo last common ancestor (Diogo, Molnar, and Wood 2017). Whether such a model exists in extant species is still debated, but it does indicate that the bonobo is a relevant piece of the evolutionary puzzle. Our previous research on the bonobo thumb revealed that their TMC joint is highly similar to that of humans, both in form and function (van Leeuwen et al. 2018, 2019). These similarities are further supported in the current study as the intra articular stress patterns encountered correspond well similar grasps tested in humans (Schneider et al. 2017). Chimpanzees also possess a similarly shaped TMC joint (Lewis 1977; Marzke et al. 2010; Rafferty 1990), likely with a similar capacity for mobility (Rose 1992), and so homologous joint

Peerj reviewing PDF | (2021:01:57152:1:1:NEW 7 Jul 2021) 
335 loading patterns may be expected. However, caution should be taken when making such 336 assumptions considering the behavioural differences between both species of Pan (Hare, 337 Wobber, and Wrangham 2012; Manson, Perry, and Parish 1997). Bonobos are reportedly more 338 arboreal (Doran 1993), while chimpanzees appear to engage in tool use more often than bonobos 339 (Neufuss et al. 2017). Yet these differences in reported locomotor and tool use behaviour may 340 also be a reflection of the offset in study frequency between these taxa and need to be studied in

341

342

343

344

345

346

347

348

349

350

351

352

353

354

355

356

357

358

359

360

361

362

363

364

365

366

367

368

369

370

371

372

373

374

375

376

377

378 detail. It would certainly be interesting to apply our FEA approach to a broader set of primate taxa, including not only the closely related chimpanzee but also primates displaying variations on the saddle-shaped TMC joint (e.g. Gorilla, Pongo, Papio, Macaca), as well as those that display a completely different joint shape (e.g. Hylobatidae) (Rafferty 1990). To that end, this study provides a suitable FEA method to investigate joint interactions and stress patterns within these morphologies for future work.

We have developed an innovative modelling workflow for the estimation of intra-articular stress distributions in a joint, and demonstrated its performance in the bonobo TMC joint. We want to stress that, rather than aiming to provide accurate estimations of stress distributions in the bonobo TMC joint, our aim was to compare stress distribution patterns between grip types and between individuals. To increase the accuracy of the model, detailed information on non-human primate tissue properties and dimensions is needed. Since such information is still lacking, we make several assumptions when building our models. First of all, a simplified cartilage layer was included in our FE model as we were unable to obtain accurate cartilage dimensions for the studied specimens and had to rely on human-based studies. Furthermore, we modelled the cartilage layer as a layer with a constant thickness, while it has been shown that cartilage varies in thickness across the articular surface in the human TMC joint, be it only slightly $(<10 \%)$ (Dourthe et al. 2019; Koff et al. 2003). In future modelling, we would recommend to include known dimensions of the cartilage layer as variations in cartilage thickness across the articular surface are likely to influence joint congruency and stress distributions. Inclusion of an accurate, nonuniform cartilage layer may affect the morphology of the TMC articular surfaces so that a more accurate representation of the stress distribution in the joint can be achieved. Our modelling approach has been designed with a modular cartilage layer to ensure that available information on TMC joint cartilage can be included in future studies. Secondly, the cartilage layer elastic properties were selected based on the previous human models (Schneider et al. 2017), and tuned within the range of human literature to the current values (Young's modulus = $18 \mathrm{MPa}$, Poisson's ratio $=0.45$ ) (Black and Hastings 2013). This to increase the robusticity of the modelling, and to ensure a higher success ratio throughout the large amount of models tested in various positions of the MC1. We expect that our choice for the parameters minimally affects the results of the study, as we compare differences in the distribution of stress, for which the exact properties have been shown to be less relevant (Gil Espert, Marcé Nogué, and Sánchez 2015).

While the sample size for this study was small due to inherent limitations in obtaining cadaver material from bonobos, a critically endangered species with a small captive population (Fruth et al. 2016), our approach allowed us to perform an in depth analysis of form-function relation surpassing the level of detail of existing studies on non-human primates. We did, however, observe some sensitivity to joint model shape during certain wide grasps in our approach. Due to

PeerJ reviewing PDF | (2021:01:57152:1:1:NEW 7 Jul 2021) 
379 this sensitivity, not all models were able to terminate successfully and we had to eliminate three 380 simulations during our analysis. Pp5's powerLA position was excluded due to severe cases of

381

382

383

384

385

386

387

388

389

390

391

392

393

394

395

396

397

398

399

400

401

402

403

404

405

406

407

408

409

410

411

412

413

414

415

416

417

418

419

420

421 edge loading in the later stages of the simulation. Edge loading occurs as a result of the lofting approach used to simulate the TMC cartilage layer, where the edge of the cartilage is artificially high compared to the surrounding bone. This mainly presents an issue during simulations of extreme MC1 excursions where the only remaining contact with the trapezium is maintained directly onto this artificial cartilage edge. Such extreme loading on the edge cause the peripheral elements of the mesh to collapse, and the simulation to fail. We furthermore had to exclude two grasp types of the sub-adult specimen Pp2. The relatively smooth morphology of the articulating bones presented difficulties in simulating grasps with an abducted thumb (powerL and powerS) and these positions could not be successfully terminated using our approach. Despite the low sample size, the open source FE method presented here is widely applicable for the study of stress distributions in joints, regardless of species or joint system.

This study yields biomechanical insight in the bonobo thumb, moreover it provides subchondral stress distributions of the TMC joint which will be included in future research on joint morphology in bonobos. The exact locations and magnitudes of these forces will be applied to investigate if the orientation of the trabecular microarchitecture of the bonobo trapezium correlates with the stress distributions estimated here. This to investigate how the bonobo thumb morphology is adapted to different types of manual activities. Moreover, it will provide in depth information that is directly relevant for the interpretation of fossil remains.

\section{Conclusions}

The present study applies a parametric FE approach to simulate stress distributions in the TMC joint of bonobos with the aim of investigating how stress distribution differs during five grasps commonly employed in captive populations. We find that the simulated stress patterns correspond well with the expected patterns during the modelled grasps, and the divergent signals can be attributed to the individual joint morphology. We conclude that inter-individual variation in joint morphology has a strong influence on intra-articular stress distributions and should always be considered when modelling joint interactions. The results demonstrate that this modelling workflow is suitable to estimate TMC joint stress distributions and can be a valuable tool for the study of primate joint biomechanics in future work.

\section{Acknowledgements}

The authors are indebted to Jeroen Stevens for his initiative taken in the collection of bonobo specimens and his courtesy in collaborating with our lab to make this study possible. We are thankful to Maarten Vanneste, Ted Yeung, Nynke Rooks, and Mousa Kazemi for their expertise and input on modelling and programming. We extend our gratitude to Olivier Vanovermeire and Henk Lacaeyse at the Medical Imaging Department, AZ Groeninge (Kortrijk, Belgium) for providing us with the means and assistance for the imaging protocol. Furthermore, we thank Marie Vanhoof and Kristiaan D'Aout for their courtesy in supplying us with primate images. The authors 
422

423

424

425

426

427

428

429

430

431

432

433

434

435

436

437

438

439

440

441

442

443

444

445

446

447

448

449

450

451

452

453

454

455

456

457

458

459

460

461

462

463

464

465

466

467

thank Johanna Neufuss, Tracy Kivell, and one anonymous reviewer for their valuable input which greatly helped with the improvement of this manuscript.

\section{References}

Bardo, Ameline, Antony Borel, Hélène Meunier, Jean Pascal Guéry, and Emmanuelle Pouydebat. 2016. "Behavioral and Functional Strategies during Tool Use Tasks in Bonobos." American Journal of Physical Anthropology 161(1):125-40.

Black, Jonathan and Garth Hastings. 2013. Handbook of Biomaterial Properties. Springer Science \& Business Media.

Burkhart, Timothy A., David M. Andrews, and Cynthia E. Dunning. 2013. "Finite Element Modeling Mesh Quality, Energy Balance and Validation Methods: A Review with Recommendations Associated with the Modeling of Bone Tissue.” Journal of Biomechanics 46(9):1477-88.

Christel, M. 1993. "Grasping Techniques and Hand Preferences in Hominoidea." Pp. 91-108 in Hands of Primates, edited by H. Preuschoft and D. J. Chivers. Vienna: Springer Vienna.

Christel, M. I., S. Kitzel, and C. Niemitz. 1998. "How Precisely Do Bonobos (Pan Paniscus) Grasp Small Objects?” International Journal of Primatology 19(1):165-93.

Cignoni, P., M. Callieri, M. Corsini, M. Dellepiane, F. Ganovelli, and G. Ranzuglia. 2008. "MeshLab: An Open-Source Mesh Processing Tool." Pp. 129-36 in Eurographics Italian Chapter Conference.

Cooney, W. P. and E. Y. Chao. 1977. "Biomechanical Analysis of Static Forces in the Thumb during Hand Function." The Journal of Bone and Joint Surgery. American Volume 59(1):27-36.

Crast, Jessica, Dorothy Fragaszy, Misato Hayashi, and Tetsuro Matsuzawa. 2009. "Dynamic InHand Movements in Adult and Young Juvenile Chimpanzees (Pan Troglodytes)." American Journal of Physical Anthropology 138(3):274-85.

Crisco, Joseph J., Eni Halilaj, Douglas C. Moore, Tarpit Patel, Arnold Peter C. Weiss, and Amy L. Ladd. 2015. "In Vivo Kinematics of the Trapeziometacarpal Joint during Thumb Extension-Flexion and Abduction-Adduction." Journal of Hand Surgery 40(2):289-96.

D’Agostino, Priscilla, Benjamin Dourthe, Faes Kerkhof, Filip Stockmans, and Evie E. Vereecke. 2017. "In Vivo Kinematics of the Thumb during Flexion and Adduction Motion: Evidence for a Screw-Home Mechanism." Journal of Orthopaedic Research 35(7):1556-64.

Diogo, Rui, Julia L. Molnar, and Bernard Wood. 2017. "Bonobo Anatomy Reveals Stasis and Mosaicism in Chimpanzee Evolution, and Supports Bonobos as the Most Appropriate Extant Model for the Common Ancestor of Chimpanzees and Humans." Scientific Reports 7(1):1-8.

Diogo, Rui, Brian Shearer, J. M. Potau, J. F. Pastor, Felix J. de Paz, Julia Arias-Martorell, Cassandra Turcotte, Ashley Hammond, Evie E. Vereecke, Marie J. M. Vanhoof, Sandra Nauwelaerts, and Bernard Wood. 2017. Photographic and Descriptive Musculoskeletal Atlas of Bonobos. 1st ed. Springer International Publishing.

Doran, Diane M. 1993. "Comparative Locomotor Behavior of Chimpanzees and Bonobos: The Influence of Morphology on Locomotion.” American Journal of Physical Anthropology 91(1):83-98.

Dourthe, Benjamin, Priscilla D’Agostino, Filip Stockmans, Faes Kerkhof, and Evie Vereecke. 2016. "In Vivo Contact Biomechanics in the Trapeziometacarpal Joint Using Finite Deformation Biphasic Theory and Mathematical Modelling.” Medical Engineering \& 
468

469

470

471

472

473

474

475

476

477

478

479

480

481

482

483

484

485

486

487

488

489

490

491

492

493

494

495

496

497

498

499

500

501

502

503

504

505

506

507

508

509

510

511

512

513

Physics 38(2):108-14.

Dourthe, Benjamin, Reza Nickmanesh, David R. Wilson, Priscilla D’Agostino, Amit N. Patwa, Mark W. Grinstaff, Brian D. Snyder, and Evie Vereecke. 2019. "Assessment of Healthy Trapeziometacarpal Cartilage Properties Using Indentation Testing and Contrast-Enhanced Computed Tomography." Clinical Biomechanics 61:181-89.

Feix, Thomas, Tracy L. Kivell, Emmanuelle Pouydebat, and Aaron M. Dollar. 2015. "Estimating Thumb-Index Finger Precision Grip and Manipulation Potential in Extant and Fossil Primates." Journal of The Royal Society Interface 12(106):20150176-20150176.

Fruth, B., J. R. Hickey, C. André, T. Furuichi, J. Hart, T. Hart, H. Kuehl, F. Maisels, J. Nackoney, G. Reinartz, T. Sop, J. Thompson, and E. A. Williamson. 2016. "Pan Paniscus." The IUCN Red List of Threatened Species 2016.

Gil Espert, L., J. Marcé Nogué, and M. Sánchez. 2015. "Insights into the Controversy over Materials Data for the Comparison of Biomechanical Performance in Vertebrate." Palaeontologia Electronica.

Haines, R. W. 1944. "The Mechanism of Rotation at the First Carpo-Metacarpal Joint.” Journal of Anatomy 78(Pt 1-2):44-46.

Halilaj, Eni, Michael J. Rainbow, Christopher J. Got, Douglas C. Moore, and Joseph J. Crisco. 2013. "A Thumb Carpometacarpal Joint Coordinate System Based on Articular Surface Geometry." Journal of Biomechanics 46(5):1031-34.

Hare, Brian, Victoria Wobber, and Richard Wrangham. 2012. "The Self-Domestication Hypothesis: Evolution of Bonobo Psychology Is Due to Selection against Aggression.” Animal Behaviour 83(3):573-85.

Ingmanson, Ellen J. 1998. "Tool-Using Behaviour in Wild Pan Paniscus: Social and Ecological Considerations.” P. 464 in Reaching Into Thought: The Minds of the Great Apes, edited by

A. E. Russon, K. A. Bard, and S. T. Parker. Cambridge University Press.

Inouye, Sandra E. 1992. "Ontogeny and Allometry of African Ape Manual Rays." Journal of Human Evolution 23(2):107-38.

Jakob, Wenzel, Marco Tarini, Daniele Panozzo, and Olga Sorkine-Hornung. 2015. "Instant Field-Aligned Meshes." ACM Transactions on Graphics 34(6):1-15.

Jordan, Claudia. 1982. "Object Manipulation and Tool-Use in Captive Pygmy Chimpanzees (Pan Paniscus)." Journal of Human Evolution 11(1):35-39.

Kerkhof, Faes D., Evie E. Vereecke, Olivier Vanovermeire, Jeroen Vanhaecke, Maarten Vanneste, and Filip Stockmans. 2018. "Trapeziometacarpal Stabilization through Dorsoradial Ligament Reconstruction: An Early Post-Surgery in Vivo Biomechanical Analyses.” Journal of Orthopaedic Research ${ }^{\circledR}$ Manuscript.

Koff, Matthew F., Obinwanne F. Ugwonali, Robert J. Strauch, Melvin P. Rosenwasser, Gerard A. Ateshian, and Van C. Mow. 2003. "Sequential Wear Patterns of the Articular Cartilage of the Thumb Carpometacarpal Joint in Osteoarthritis." The Journal of Hand Surgery 28(4):597-604.

Kuczynski, K. 1974. "Carpometacarpal Joint of the Human Thumb.” Journal of Anatomy 118(Pt 1):119-26.

Ladd, Amy L., Joseph J. Crisco, Elisabet Hagert, Jessica Rose, and Arnold-Peter C. Weiss. 2014. "The 2014 ABJS Nicolas Andry Award: The Puzzle of the Thumb: Mobility, Stability, and Demands in Opposition." Clinical Orthopaedics and Related Research ${ }^{\circledR}$ 472(12):3605-22.

Ladd, Amy L., Arnold-Peter C. Weiss, Joseph J. Crisco, Elisabet Hagert, Jennifer Moriatis Wolf, Steven Z. Glickel, and Jeffrey Yao. 2013. "The Thumb Carpometacarpal Joint: Anatomy, 
514

515

516

517

518

519

520

521

522

523

524

525

526

527

528

529

530

531

532

533

534

535

536

537

538

539

540

541

542

543

544

545

546

547

548

549

550

551

552

553

554

555

556

557

558

559

Hormones, and Biomechanics." Instructional Course Lectures 62:165-79.

van Leeuwen, Timo, Marie J. M. Vanhoof, Faes D. Kerkhof, Jeroen M. G. Stevens, and Evie E. Vereecke. 2018. "Insights into the Musculature of the Bonobo Hand." Journal of Anatomy 233(3):328-40.

van Leeuwen, Timo, Maarten Vanneste, Faes D. Kerkhof, Priscilla D’agostino, Marie J. M. Vanhoof, Jeroen M. G. Stevens, G. Harry van Lenthe, and Evie E. Vereecke. 2019. "Mobility and Structural Constraints of the Bonobo Trapeziometacarpal Joint." Biological Journal of the Linnean Society 127(3):681-93.

Lewis, O. J. 1977. "Joint Remodelling and the Evolution of the Human Hand." Journal of Anatomy 123(Pt 1):157-201.

Maas, Steve A., Benjamin J. Ellis, Gerard A. Ateshian, and Jeffrey A. Weiss. 2012. "FEBio: Finite Elements for Biomechanics." Journal of Biomechanical Engineering 134(1):011005.

Manson, Joseph H., Susan Perry, and Amy R. Parish. 1997. "Nonconceptive Sexual Behavior in Bonobos and Capuchins.” International Journal of Primatology 18(5):767-86.

Marzke, M. W., M. W. Tocheri, B. Steinberg, J. D. Femiani, S. P. Reece, R. L. Linscheid, C. M. Orr, and R. F. Marzke. 2010. "Comparative 3D Quantitative Analyses of

Trapeziometacarpal Joint Surface Curvatures among Living Catarrhines and Fossil Hominins." American Journal of Physical Anthropology 141(1):38-51.

Marzke, Mary W. 1997. "Precision Grips, Hand Morphology, and Tools." American Journal of Physical Anthropology 102(1):91-110.

Miller, Ruth A. 1952. "The Musculature of Pan Paniscus." American Journal of Anatomy 91(2):183-232.

Myatt, Julia P., Robin H. Crompton, Rachel C. Payne-Davis, Evie E. Vereecke, Karin Isler, Russell Savage, Kristiaan D’Août, Michael M. Günther, and Susannah K. S. Thorpe. 2012. "Functional Adaptations in the Forelimb Muscles of Non-Human Great Apes." Journal of Anatomy 220(1):13-28.

Napier, J. R. 1955. "The Form and Function of the Carpo-Metacarpal Joint of the Thumb." Journal of Anatomy 89(3):362-69.

Napier, J. R. 1956. "The Prehensile Movements of the Human Hand." The Journal of Bone and Joint Surgery. British Volume 38-B(4):902-13.

Napier, J. R. 1960. "Studies of the Hands of Living Primates." Proceedings of the Zoological Society of London 134:647-57.

Napier, J. R. and P. H. Napier. 1967. : : A Handbook of Living Primates: Morphology, Ecology and Behaviour of Nonhuman Primates. Academic P.

Neufuss, Johanna, Tatyana Humle, Andrea Cremaschi, and Tracy L. Kivell. 2017. "NutCracking Behaviour in Wild-Born, Rehabilitated Bonobos (Pan Paniscus): A Comprehensive Study of Hand-Preference, Hand Grips and Efficiency." American Journal of Primatology 79(2):1-16.

Parker, Sue Taylor and Kathleen R. Gibson. 1977. "Object Manipulation, Tool Use and Sensorimotor Intelligence as Feeding Adaptations in Cebus Monkeys and Great Apes." Journal of Human Evolution 6(7):623-41.

Payne, R. C., R. H. Crompton, K. Isler, R. Savage, E. E. Vereecke, M. M. G??nther, S. K. S. Thorpe, and K. D'Ao??t. 2006. "Morphological Analysis of the Hindlimb in Apes and Humans. II. Moment Arms." Journal of Anatomy 208(6):725-42.

Payne, R. C., R. H. Crompton, K. Isler, R. Savage, E. E. Vereecke, M. M. Günther, S. K. S. Thorpe, and K. D'Août. 2006. "Morphological Analysis of the Hindlimb in Apes and

PeerJ reviewing PDF | (2021:01:57152:1:1:NEW 7 Jul 2021) 
560

561

562

563

564

565

566

567

568

569

570

571

572

573

574

575

576

577

578

579

580

581

582

583

584

585

586

587

588

589

590

591

592

593

594

595

596

597

598

599

600

Humans. I. Muscle Architecture.” Journal of Anatomy 208(6):709-24.

Prüfer, Kay, Kasper Munch, Ines Hellmann, Keiko Akagi, Jason R. Miller, Brian Walenz, Sergey Koren, Granger Sutton, Chinnappa Kodira, Roger Winer, James R. Knight, James C. Mullikin, Stephen J. Meader, Chris P. Ponting, Gerton Lunter, Saneyuki Higashino, Asger Hobolth, Julien Dutheil, Emre Karakoç, Can Alkan, Saba Sajjadian, Claudia Rita Catacchio, Mario Ventura, Tomas Marques-Bonet, Evan E. Eichler, Claudine André, Rebeca Atencia, Lawrence Mugisha, Jörg Junhold, Nick Patterson, Michael Siebauer, Jeffrey M. Good, Anne Fischer, Susan E. Ptak, Michael Lachmann, David E. Symer, Thomas Mailund, Mikkel H. Schierup, Aida M. Andrés, Janet Kelso, and Svante Pääbo. 2012. "The Bonobo Genome Compared with the Chimpanzee and Human Genomes." Nature 486(7404):52731.

Rafferty, Katherine L. 1990. "The Functional and Phylogenetic Significance of the Carpometacarpal Joint of the Thumb in Anthropoid Primates." New York University.

Rose, M. D. 1992. "Kinematics of the Trapezium-1st Metacarpal Joint in Extant Anthropoids and Miocene Hominoids." Journal of Human Evolution 22(4-5):255-66.

Samuel, Diana S., Sandra Nauwelaerts, Jeroen M. G. Stevens, and Tracy L. Kivell. 2018. "Hand Pressures during Arboreal Locomotion in Captive Bonobos ( Pan Paniscus )." The Journal of Experimental Biology 221(8):jeb170910.

Schneider, M. T. Y., J. Zhang, J. J. Crisco, A. P. C. Weiss, A. L. Ladd, P. Nielsen, and T. Besier. 2015. "Men and Women Have Similarly Shaped Carpometacarpal Joint Bones." Journal of Biomechanics 48(12):3420-26.

Schneider, Marco T. Y., Ju Zhang, Joseph J. Crisco, Arnold-Peter C. Weiss, Amy L. Ladd, Kumar Mithraratne, Poul Nielsen, and Thor Besier. 2017. "Trapeziometacarpal Joint Contact Varies between Men and Women during Three Isometric Functional Tasks." Medical Engineering \& Physics 50:43-49.

Simpson, Scott W., Bruce Latimer, and C. Owen Lovejoy. 2018. "Why Do Knuckle-Walking African Apes Knuckle-Walk?” The Anatomical Record 301(3):496-514.

Susman, Randall L. 1998. "Hand Function and Tool Behavior in Early Hominids." Journal of Human Evolution 35(1):23-46.

Tuttle, Russell H. 1967. "Knuckle-Walking and the Evolution of Hominoid Hands." American Journal of Physical Anthropology 26(2):171-206.

Vereecke, Evie E., Kristiaan D'Août, Rachel Payne, and Peter Aerts. 2005. "Functional Analysis of the Foot and Ankle Myology of Gibbons and Bonobos." Journal of Anatomy 206(5):45376.

Wilson, David R., Emily J. McWalter, and James D. Johnston. 2008. "The Measurement of Joint Mechanics and Their Role in Osteoarthritis Genesis and Progression." Rheumatic Disease Clinics of North America 34(3):605-22.

Wunderlich, R. E. and W. L. Jungers. 2009. "Manual Digital Pressures during Knuckle-Walking in Chimpanzees ( Pan Troglodytes )." American Journal of Physical Anthropology 139(3):394-403. 


\section{Table $\mathbf{1}$ (on next page)}

Specimen information

*Master specimen, **Scanned in 4 positions only, ***Wild born but raised in captivity, SB:

studbook number, M: male, F: female, R: right, L: left. 
1 Table 1: Specimen information

\begin{tabular}{|l|l|l|l|l|l|l|}
\hline Code & Subject ID & Age & Sex & Sample & Origin & Zoo \\
\hline Pp1 & 8365526 & 35 & M & L & Wild born & Wilhelma Zoo, Stuttgart, Germany \\
\hline Pp2 & $\begin{array}{l}15295295 \\
\text { SB: } 295\end{array}$ & 8 & F & L & Captive born & $\begin{array}{l}\text { Royal Zoological Society Antwerp, } \\
\text { Belgium }\end{array}$ \\
\hline Pp3 & $\begin{array}{l}\text { MIG12- } \\
29882197 \\
\text { SB: } 126\end{array}$ & 32 & M & R & Captive born & Zoo Frankfurt, Germany \\
\hline Pp4* & $\begin{array}{l}\text { SB:177 } \\
\text { CITES:79209 }\end{array}$ & 24 & M & R & Captive born & $\begin{array}{l}\text { La Vallée des Singes, Le Gureau, } \\
\text { France }\end{array}$ \\
\hline Pp5** & $\begin{array}{l}\text { SB: } 88 \\
27641621\end{array}$ & 39 & F & L & Wild born & Wilhelma Zoo, Stuttgart, Germany \\
\hline
\end{tabular}

2 


\section{Figure 1}

The bonobo trapeziometacarpal (TMC) joint, from a ventral (palmar) view $(A)$ and a radiodistal view $(B)$.

Its reciprocal surfaces are saddle-shaped. The distal articular surface of the trapezium (T) has a convex curvature along the dorsal-palmar (D-V) axis, and makes contact with the concave curvature of the first metacarpal (MC1), while the trapezial articular surface along the radioulnar ( $\mathbf{R}-\mathbf{U})$ axis is concave, to match the convex curvature of the $\mathrm{MCl}$. The principal movements of the thumb align with rotation about one of three axes of the joint; rotation about the $\mathbf{D}-\mathbf{V}$ axis results in palmar abduction-adduction, rotation about the $\mathbf{R}-\mathbf{U}$ axis results in thumb flexion-extension, and rotation about the proximal-distal (P-D) axis results in axial rotation of the thumb. The depicted bone models are trapezium and MC1 surface meshes reconstructed from CT-scanning Pp5 in a neutral position of the thumb.

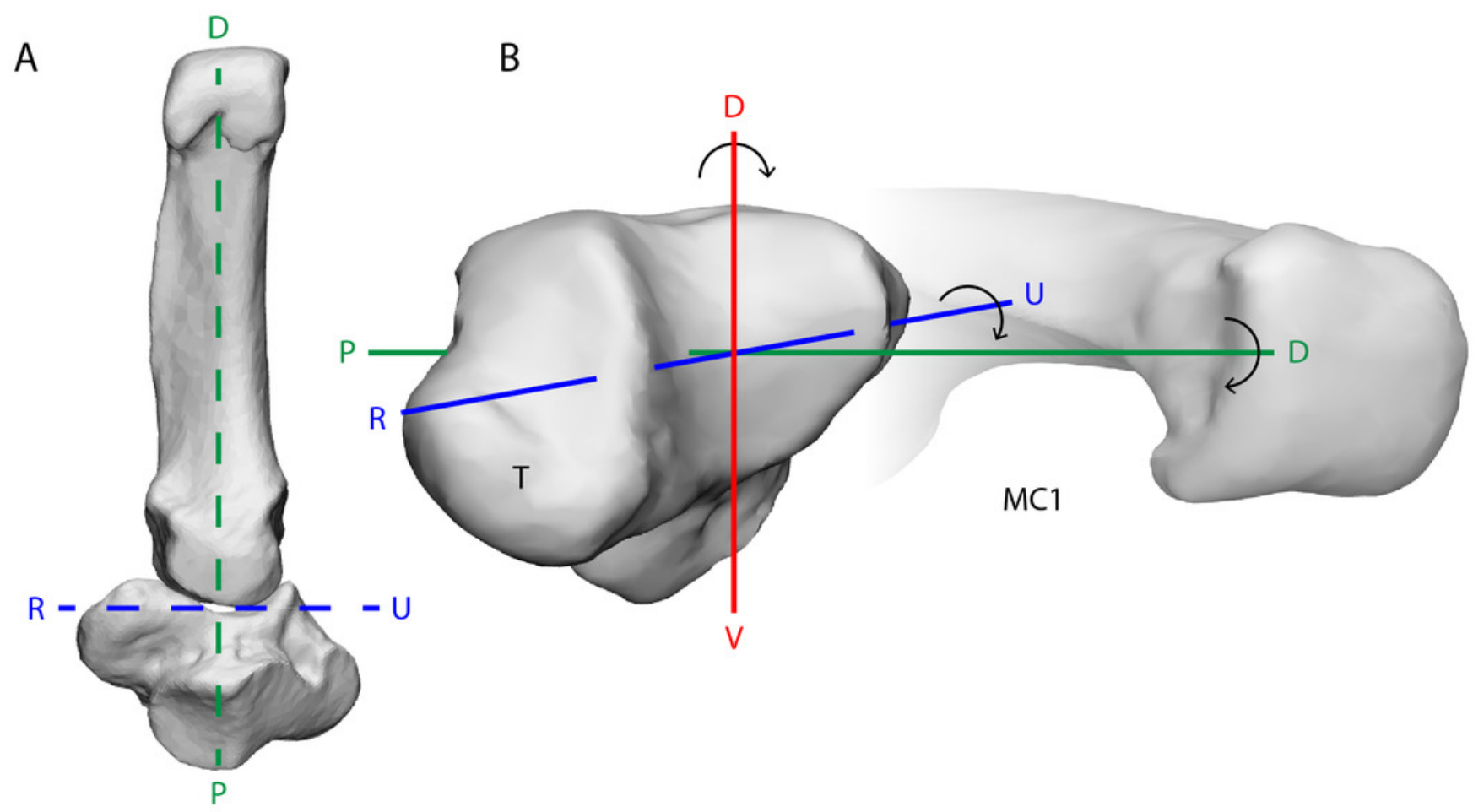




\section{Figure 2}

Depiction of the different grasping types observed in bonobos.

A: an adult male bonobo, B: large diameter power grip with thumb abducted (powerL), C: large diameter power grip with thumb extended (powerLE), D: large diameter power grip with thumb abducted (powerLA), E: small diameter power grip (powerS), F: pad-to-side precision grip or keypinch (pinch). Photography by courtesy of Marie Vanhoof and prof. Kristiaan D'Aout.

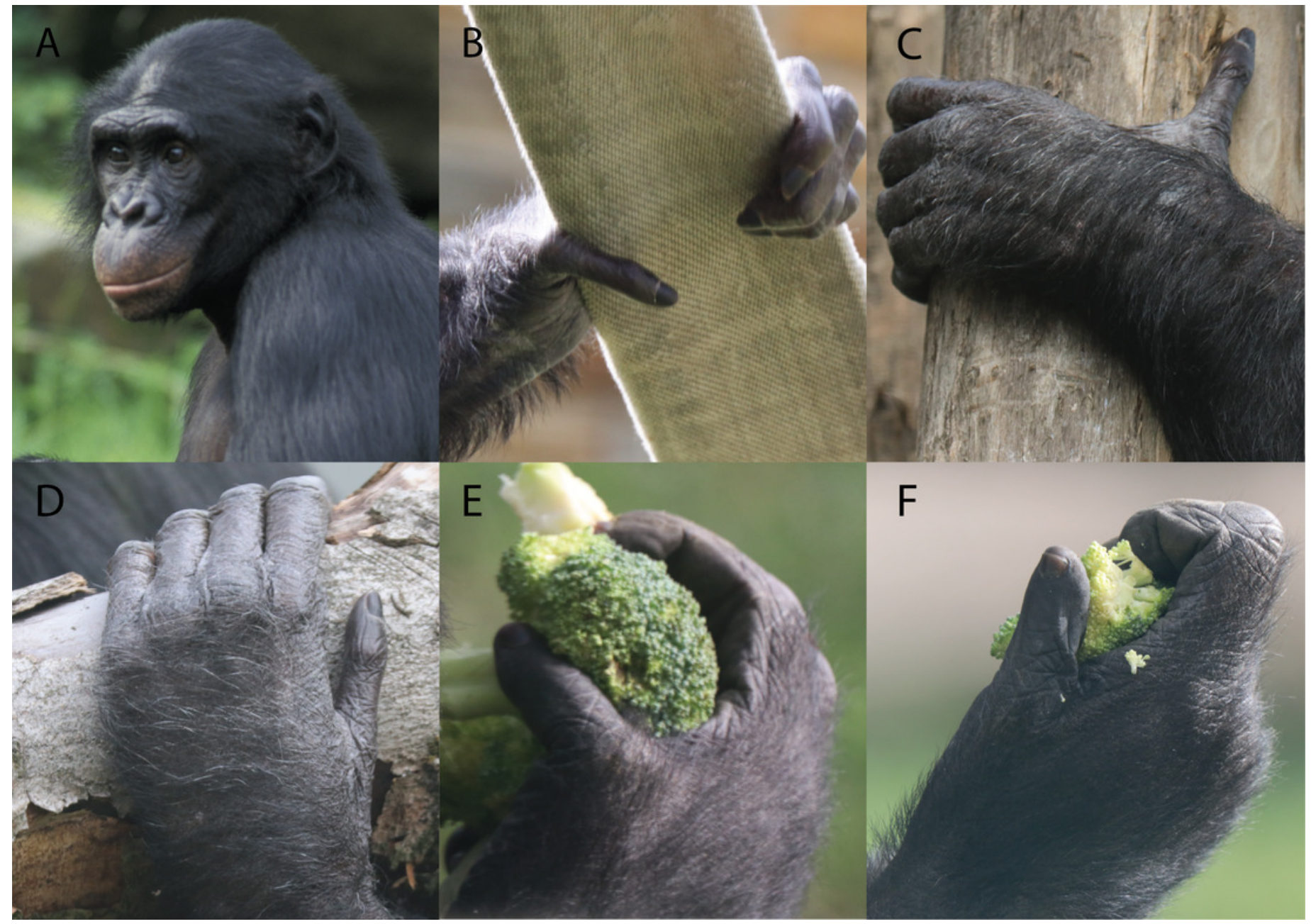




\section{Figure 3}

A schematic representation of the parametric finite element model generation pipeline

Template TMC meshes are selected for each specimen, one set is designated as the master mesh (A). All template meshes are aligned with the master mesh using rigid body registration of the trapezia, and transforming the $\mathrm{MC1}$ s onto the master meshes (B). The remaining $\mathrm{MCl}$ positions for each specimen are aligned with their corresponding template set, again using rigid body registration of the trapezia and transforming the $\mathrm{MCls}$ (C). The master template mesh is discretized into quadrilateral meshes (D), the articular surfaces are identified, extracted as quadrilateral meshes, and lofted into hexahedral cartilage meshes (not visualized). The quadrilateral bone and hexahedral cartilage (not visualized) master templates are fitted to the morphology of each specimen's template set using Radial Basis Function (RBF) fitting (E). The master $\mathrm{MC1}$ template meshes are fitted to the remaining grasping positions in each specimen using rigid body registration $(\mathbf{F})$. The workflow yields discretized quadrilateral meshes for all five specimens, for each of the five (four in the case of Pp5) positions (G). 

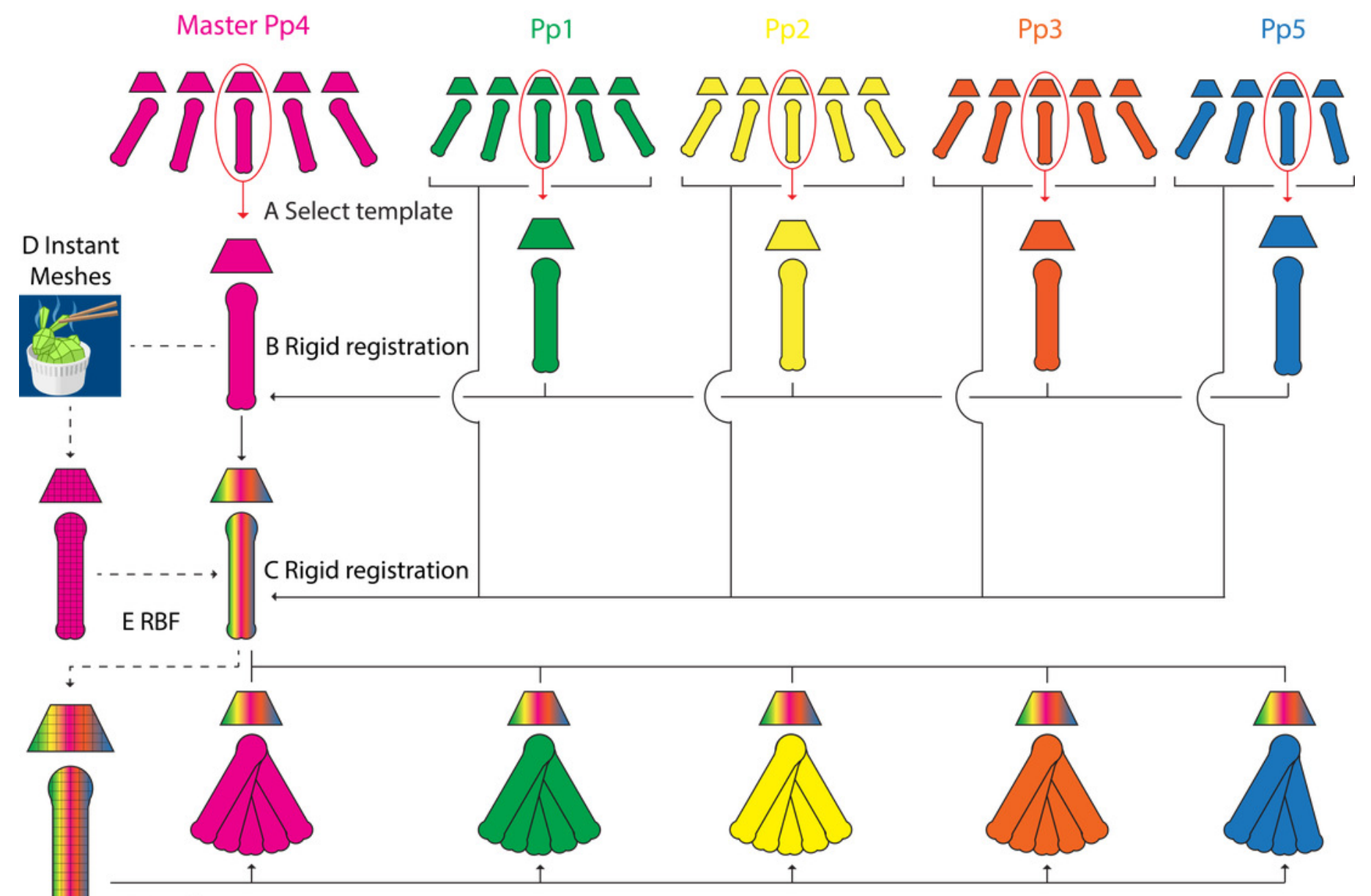

F Rigid registration

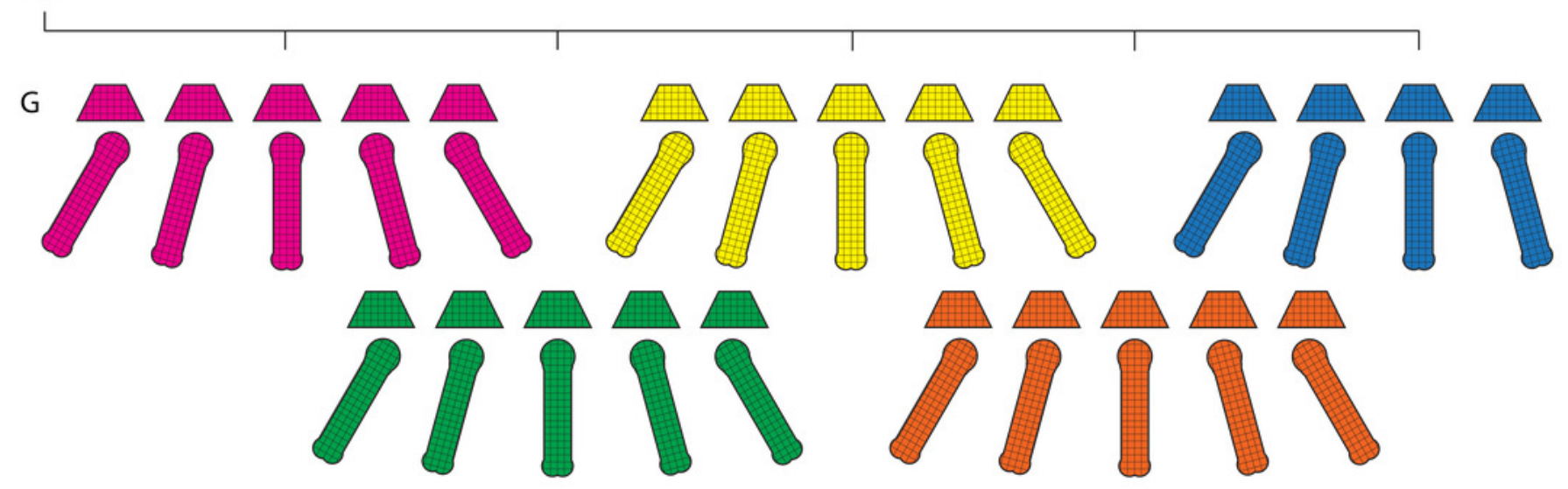

$\square=$ Trapezium $\square=$ First metacarpal $\square=$ STL mesh $\square=$ Quad mesh $\square=$ Aligned meshes 


\section{Figure 4}

The TMC joint FE model in the large diameter power grasp with extended thumb (powerLE).

The coloured surfaces represent the cartilage layers on the trapezium (pink) and MC1 (blue), black lines indicate non-linear springs and correspond to ligament positions. Shown here is the FE model based on the morphology of Pp4.

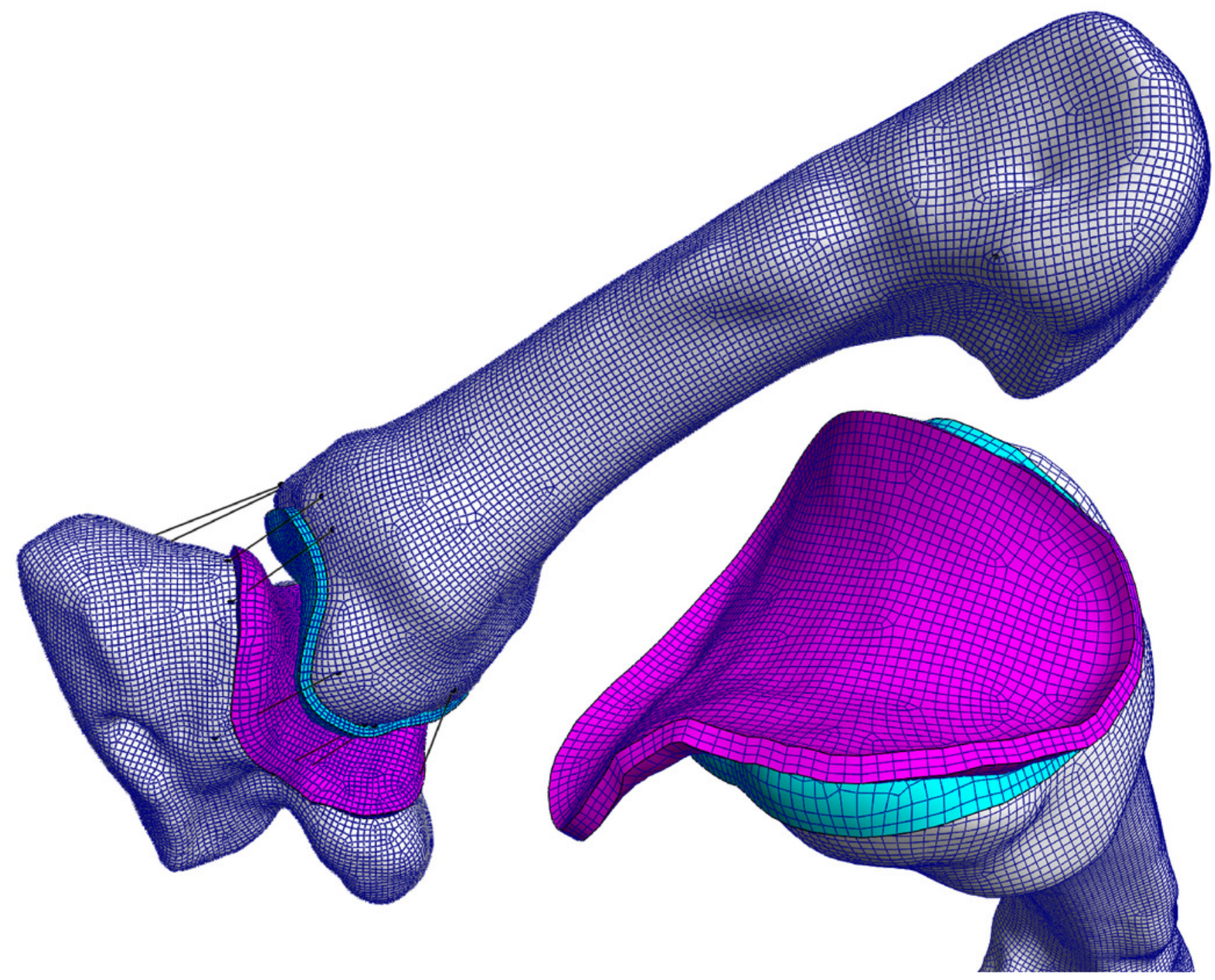




\section{Figure 5}

TMC joint Von Mises stress distributions plotted on a distal view of the isolated trapezium distal articular surfaces

The top row displays the quadrants where stress distribution is expected for each position in red. Empty slots represent unsuccessful scans or simulations.
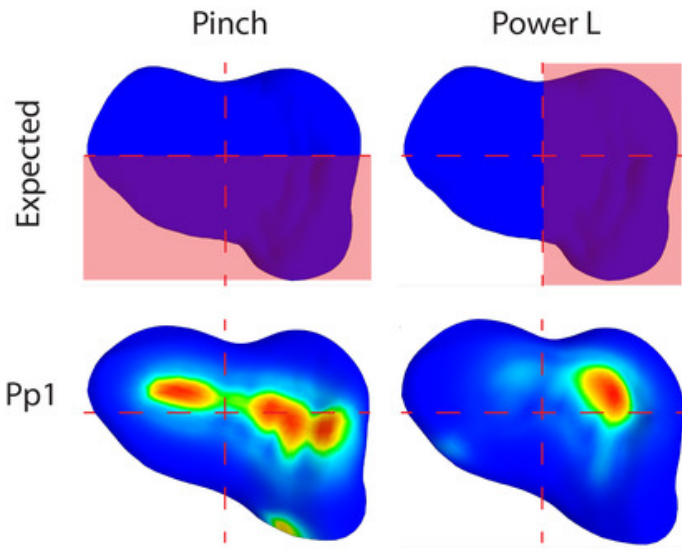

Pp2
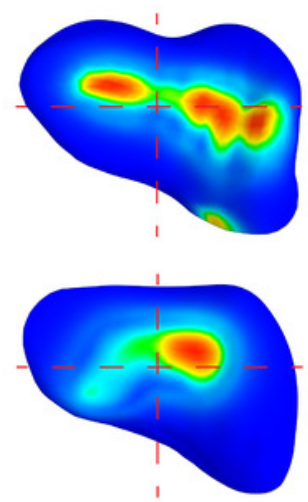

$\operatorname{Pp} 3$
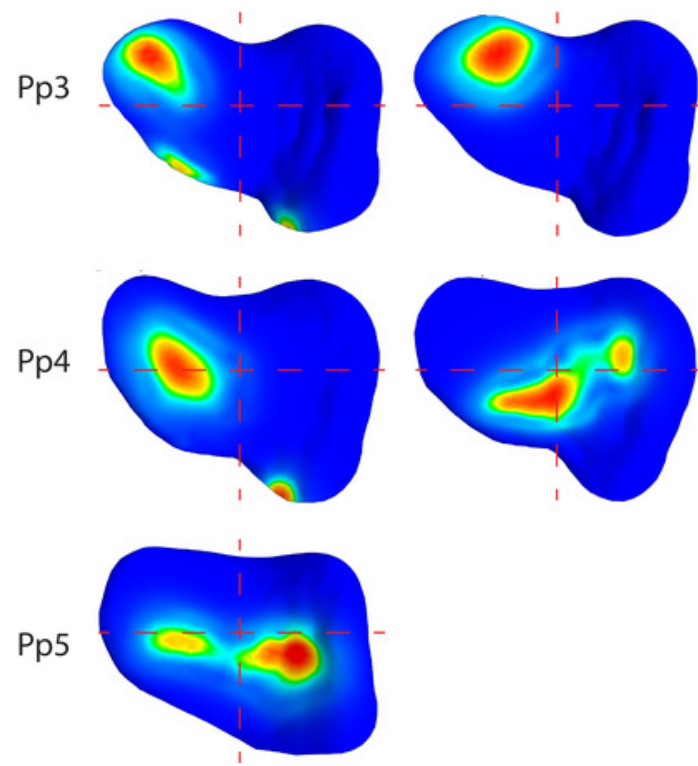

Power LA
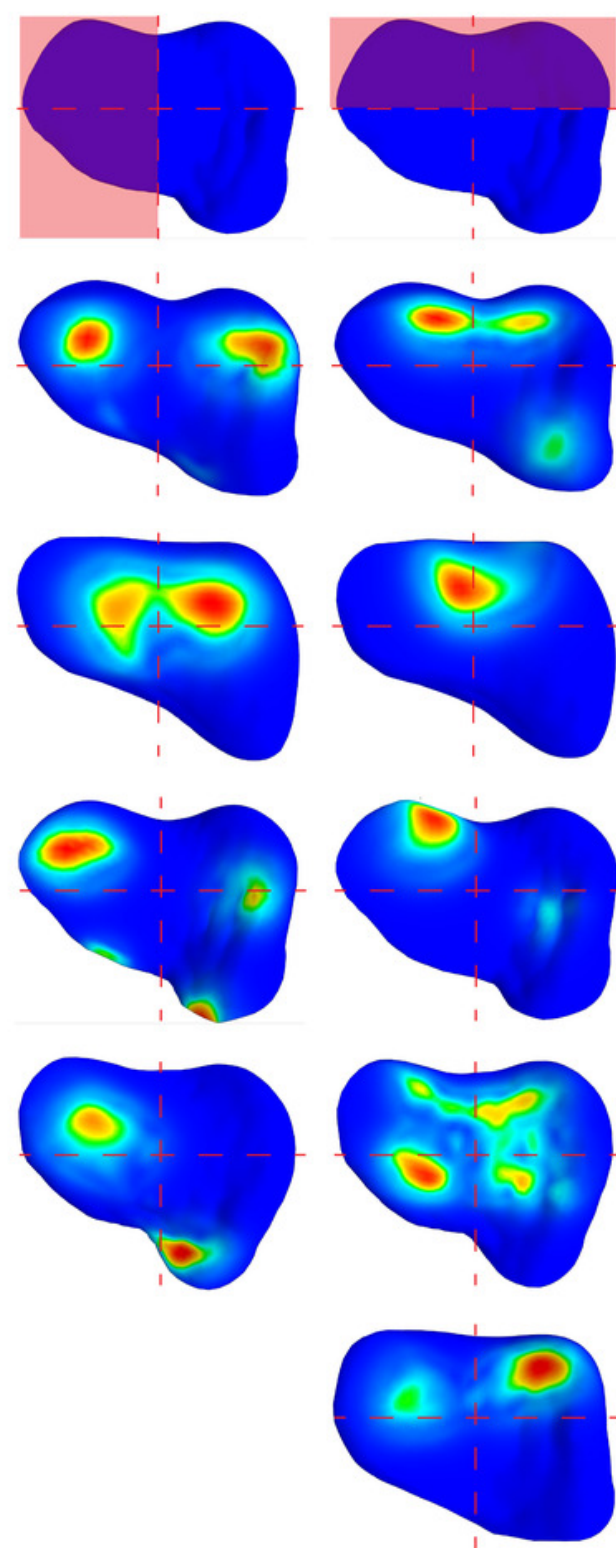

Power S
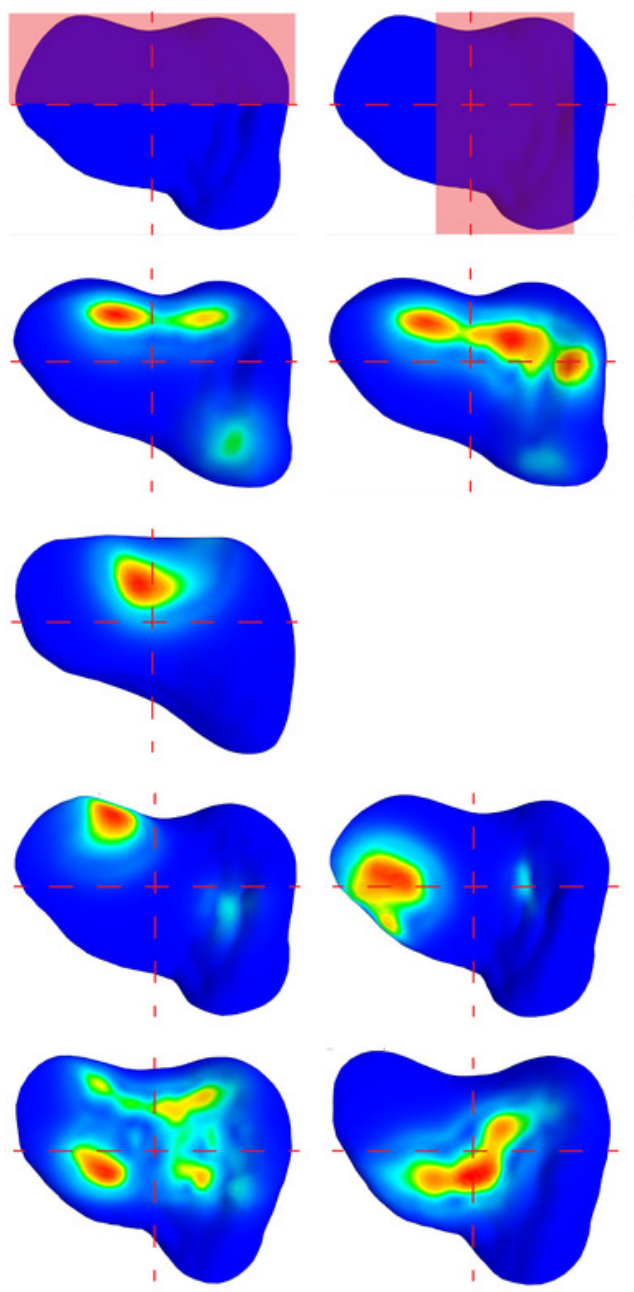

Von Mises

Stress (MPa)

High

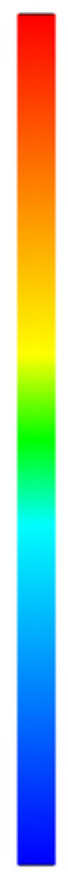

Low

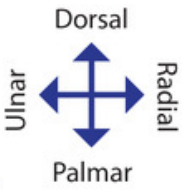




\section{Figure 6}

Deviating morphologies in specimens Pp1-4 in the top row in comparison to more common morphologies of the remaining specimens in the bottom row

A: Radial view of the protruding dorsal ridge and volar beak of Pp1's MC1 base. B: Dorsal view of the asymmetrical dorsal ridge of Pp2's MC1 base. C: Dorsal view of Pp3's protruding dorso-ulnar periphery of the trapezial articular facet. D: Disto-ulnar view of the Pp4's flattened palmar trapezial articular facet, as well as the palmar indentures highlighted in red.
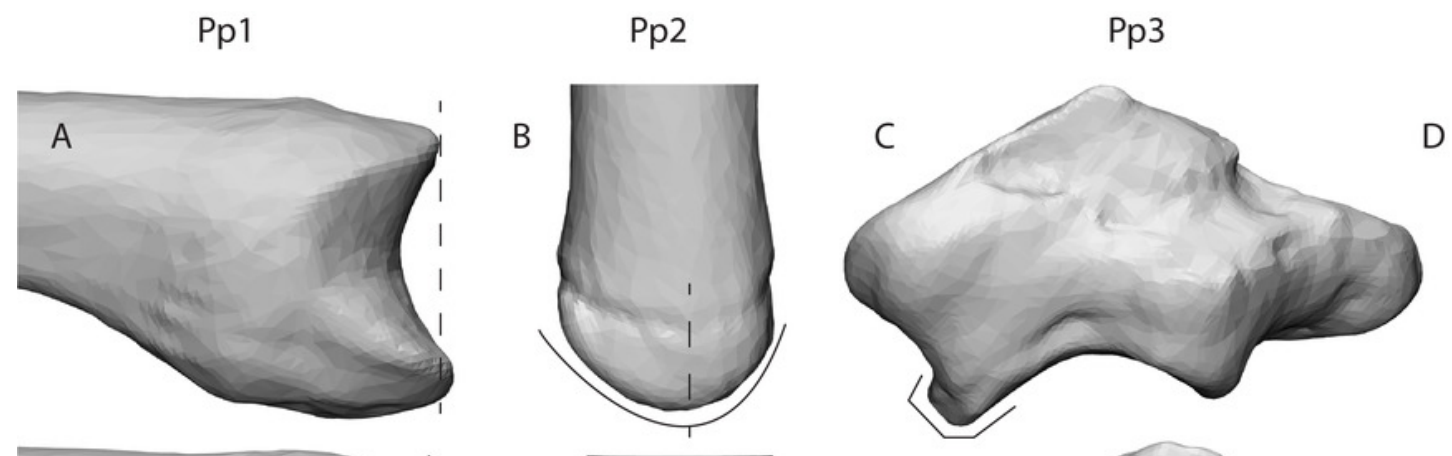

Pp4
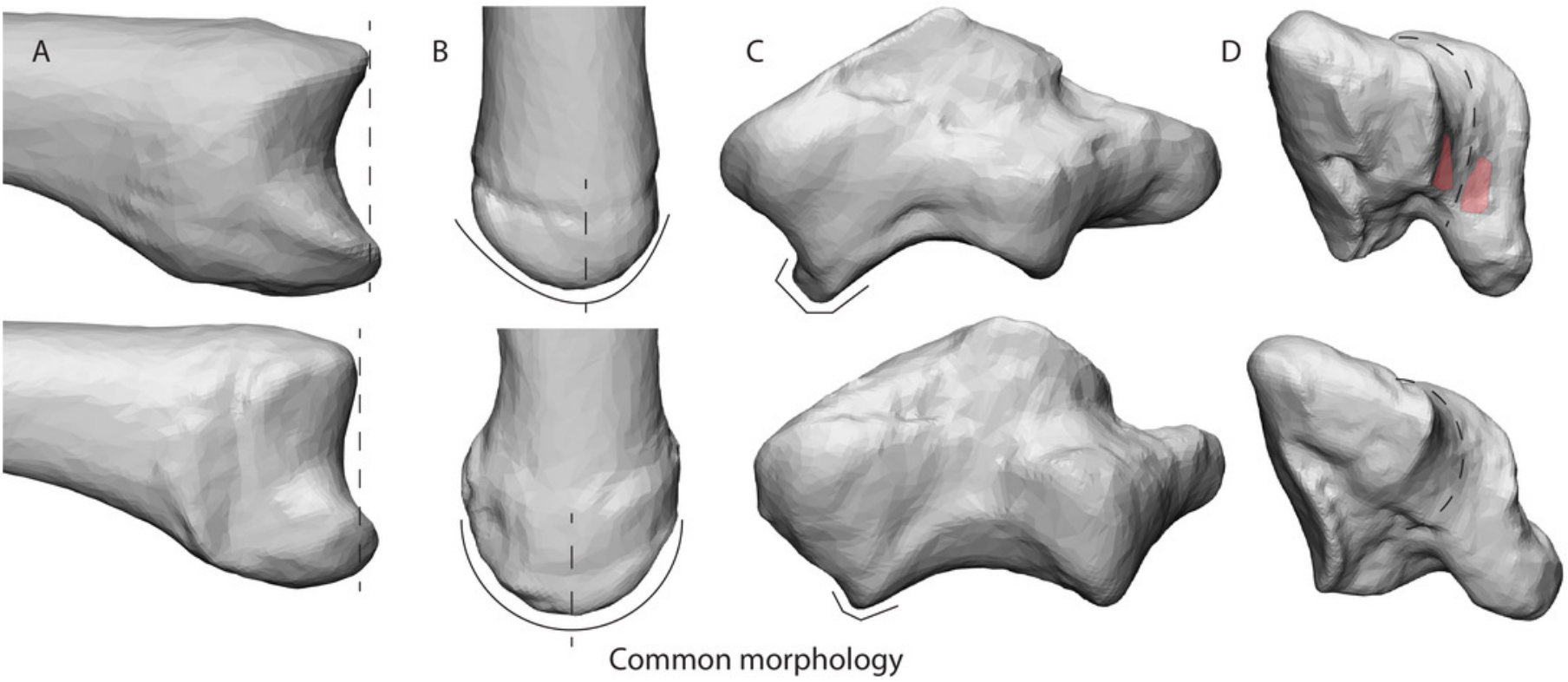